\title{
L'enjeu du sujet
}

\author{
Carole Massé
}

\section{The Subject at Stake:}

How to inscribe the feminine subject in discourse and the dominant ideology without being inscribed in its norms? These are the stakes in the game played by/with the subject. Writing exceeds and abolishes norms. The way to inscribe a subject in the feminine in texts is by passing through the subject. Withdraw from the collectivity into intimate works where what is at stake is the re-creation of the ' $I$ '. This involves an engagement of the subject in the unconscious and in language, exploring one's phantasms, one's desire, for a jouissance in writing that breaks with memory and the community. To move beyond.

Comment inscrire le sujet féminin dans le discours et l'idéologie dominante? La question n'est pas jeune. Elle nous hante encore, après tant d'années, parce que personne n'a fourni de réponse satisfaisante. Heureusement. Cette impossibilité de clore la question est garante de notre liberté.

La question s'est déjà posée par rapport à la littérature: comment inscrire le sujet féminin dans l'écriture? J'ai la conviction que répondre à cette question une fois pour toutes et pour toutes les femmes soumettrait le sujet féminin à des muliples règles et contraintes. Alors j'ose ma question: comment moi, écrivaine, puis-je m'inscrire dans la langue sans $m$ 'inscrire dans une norme?

A mon avis, écrire implique l'abolition de toute norme. Chercher les frontières ou les fondements d'une écriture féminine, féministe ou d'une écriture-femme, c'est peut-être chercher d'autres limites pour nous contenir, nous refouler et nous censurer nous-mêmes. Je formule ce voeu: aucune limite à l'imaginaire des femmes dans la fiction, aucun rôle édifiant ou répréhensible à y construire ou déconstruire pour le bien de toutes, aucune voie traçée d'avance sur papier qui mène à un destin de femme idéal. La culpabilité à inventer hors des sentiers balisés a fait son temps. 
Pour qu'advienne le sujet féminin dans des textes? Passer par le sujet, justement. Et la question d'ordre général ne s'efface-t-elle pas dans le surgissement du particulier? Passer par le sujet: position guère admissible pour toute Volonté normalisante ou Interprétation globalisante du monde. Pourtant aucune philosophie ne peut soustraire chaque sujet, femme ou homme, à sa différence inaliénable et acquitter sa dette finale: sa vie. Passer par le sujet, dans l'écriture, signifie en dernière instance se retirer du collectif et faire oeuvre intimiste dont l'enjeu est la re-création de soi.

On ne peut séparer la question du sujet de la question de l'inconscient. Je crois que la liberté totale d'explorer mes phantasmes dans l'écriture est indissociable de ma liberté d'exister en tant que femme. $\mathrm{Ne}$ jamais perdre de vue le rapport du sujet à la langue car le monde n'existe pas en dehors de ce rapport. Et le sujet sera toujours la matière privilégiée de sa propre écriture.

L'idéologie et la théorie, se nourrissant parfois de l'angoisse de l'individu face au vide ou à l'infini, tentent de quadriller l'activité créatrice. On veut baliser les méandres de l'expérience intérieure, or cette dernière échappe à la rationalisation, à l'utilitarisme et aux consensus de groupes. L'écriture est l'affirmation irréductible d'une parole qu'on ne saurait astreindre à un Cause, un Sens ou à Morale strictement revendicatrice. Elle est la demande de reconnaissance inlassable mais contradictoire du sujet: reconnaissance du nom qu'il porte et de l'innommable qui l'habite.

Proposer un modèle d'écriture aux femmes ne peut que brimer leur liberté de se concevoir et de s'écrire hors-code, hors-norme, et de se jouer multiples dans la fiction: écrivaines sans origine et sans loi, baroques, obscènes, sacrilèges, orphelines, en un mot, inimitables parce que ni maîtres ni esclaves. Travailler à l'élaboration d'une écriture 'consciente' de femme risque de bloquer l'émergence des écritures de désir. Pour ma part, m'abandonner aux seules pulsions et passions qui trament ma perte dans l'écriture s'avère l'objet de ma quête.

La force d'une oeuvre réside dans sa singularité, dans la dissonnance qu'elle constitue au coeur d'une littérature de divertissement ou de conditionnement social. La pertinence d'une signature découle de son pouvoir de raturer les effets du Nombre (par opposition à $1^{\prime} U n$ ), du Même et du mièvre érigés en culture de masse.

La contribution inestimable du mouvement des femmes? Rendre à 


\section{L'enjeu du sujet · 77}

chaque femme le pouvoir de se penser en dehors de la norme et du modèle, et de se dire dans sa propre mouvance intérieure. Car le paradoxe n'est pas le moindre. Désirer qu'une femme prenne sa place de sujet (et ne soit plus remise à 'sa' place par quelque idéologie que ce soit), c'est accepter que cette femme puisse rejeter toute allégeance ou étiquette et ne désirer plus qu'être, non pas réduite, mais agrandie à sa plus simple expression: une, dépouillée de tous points de ressemblance aux autres, revêtue de sa stricte unicité dans l'espèce humaine.

Changer de place, pour une femme, passer de la position d'objet du discours à celle de sujet dans l'écriture, signifie accéder à l'aire de sa solitude radicale et aux vertiges de son propre corps. Alors la jouissance de l'Autre et par l'Autre est tout près, la jouissance pour laquelle l'exil de l'histoire familiale et sociale s'érige en impératif. La jouissance dans le corps et celle dans l'écriture rallient étrangement les mêmes exigence de ruptures avec la mémoire et la communauté.

Mais il n'y a pas que le sujet qui soit appelé, dans la recherche du bonheur, à faire des deuils. Nous savons aujourd'hui, après l'échec de grandes idéologies, que les groupes et les mouvements sont appelés eux aussi aux mêmes dépassements. 